\title{
Avaliação isocinética no joelho do atleta
}

\author{
Antonio Sérgio A.P. Terreri, Júlia M.D. Greve e Marco M. Amatuzzi \\ Laboratório de Estudos do Movimento - Grupo de Medicina Esportiva - Instituto de Ortopedia e \\ Traumatologia do Hospital das Clínicas da Faculdade de Medicina da Universidade de São Paulo
}

\section{RESUMO}

A avaliação isocinética tem sido usada nas últimas três décadas como método para se determinar o padrão funcional da força e do equilíbrio muscular. No nosso meio cresceu nos últimos 10 anos. É possível quantificar valores absolutos do torque, do trabalho e da potência de grupos musculares, bem como valores relativos, ou seja, da proporção agonista/antagonista de tais grupos. Outro recurso valioso consiste como método auxiliar na reabilitação das lesões esportivas. O exame é realizado com velocidade angular constante e predeterminada, seja lenta, intermediária e/ou rápida. $\mathrm{O}$ joelho é a articulação em que se observa maior aplicação e estudos isocinéticos. Na prática esportiva, temos por um lado a importância da proporção do equilíbrio muscular agonista/antagonista, ou seja, do equilíbrio flexor/extensor representado, respectivamente, pelos isquiotibiais/quadríceps. De outro, a comparação dos valores absolutos da função muscular entre os lados direito e esquerdo, quer seja para o quadríceps, ou para os isquiotibiais. Resultados alterados estão relacionados, geralmente, às lesões esportivas ou suas seqüelas. De interesse para o atleta e para a equipe multidisciplinar é poder dispor de um método de avaliação funcional muscular objetivo e seguro, que forneça dados confiáveis e reprodutíveis. Aspecto relevante reside no fato do resultado do teste isocinético poder ser útil nos critérios de retorno a atividade esportiva pós-lesão. Este artigo de revisão consiste em abordar alguns aspectos práticos da avaliação isocinética no joelho e sua utilização para os indivíduos que realizam atividade física.

Palavras-chave: Isocinético. Avaliação isocinética. Joelho. Medicina esportiva. Equilíbrio muscular.

Recebido em: 25/7/2000

Aceito em: 28/8/2001

Endereço para correspondência:

Antonio Sérgio A.P. Terreri

Rua Loefgreen, 2.381 - Ap. 141

04040-004 - São Paulo, SP

E-mail: terreri@uninet.com.br

\section{ABSTRACT}

\section{Isokinetic evaluation of athletes' knee}

The isokinetic evaluation has been used in the last three decades as a method to determine both the strength functional pattern and the muscle balance. In our environment, this evaluation has been spread over the last 10 years. It is possible to quantify absolute values of torque, effort, and power of muscle groups, and to determine relative values, that is, the agonist/antagonist proportion of such groups. Another valuable resource of this evaluation may be used as an auxiliary method in the rehabilitation of sports lesions. The examination is performed with a constant and preset angular speed, that is, low, intermediate, and/or high speed. Most isokinetic studies are applied to the knee. In the sports practice, the agonist/antagonist muscle balance, that is, the flexor/extensor balance represented, respective$l y$, by the ischiotibial musclelquadriceps plays an important role. On the other hand, the comparison between the absolute values of the muscle function for the left and right sides is also relevant. Changed results usually relate to sports lesions or their sequels. The development of an objective and safe method to evaluate the muscle functionality which provides for reliable and reproducible results is of utmost importance to athletes and to multidisciplinary teams. Also significant are the results obtained from isokinetic tests, which may be useful to establish criteria for athletes to resume and practice sports activities after lesions. This review aims at addressing some practical aspects of the isokinetic evaluation in the knee and its use in individuals performing sports activities.

Key words: Isokinetics. Isokinetic evaluation. Knee. Sports medicine. Muscle balance.

\section{INTRODUÇÃO}

O conceito de equilíbrio funcional musculotendíneo reflete um parâmetro importante na adequada realização da prática de esportes. A avaliação isocinética tem sido usada nas últimas três décadas como método para se determinar o padrão funcional da força e do equilíbrio muscular. Em- 
bora o termo isocinético tenha sido já definido em 1967², seu uso tornou-se mais expressivo nas últimas duas décadas. No nosso meio, a experiência tem sido crescente nos últimos 10 anos $^{2-5}$.

O aparelho isocinético é um dinamômetro eletromecânico com sistema servomotor, que atualmente se apresenta todo computadorizado. O indivíduo realiza um esforço muscular máximo ou submáximo que se acomoda à resistência do aparelho. Este se caracteriza por possuir velocidade angular constante, permitindo realizar movimento na sua amplitude articular. A força exercida pelos grupos musculares varia durante o arco de movimento, devido ao seu braço de alavanca que se altera conforme a amplitude do movimento. Tem-se, então, o chamado momento angular de força ou torque. A resistência oferecida também é variável conforme a força realizada em cada ponto da amplitude articular. Mas a velocidade angular é sempre constante, em graus por segundo $(\% / \mathrm{seg})$, definida previamente pelo examinador ${ }^{6}$.

O aparelho isocinético é um recurso valioso, podendo ser indicado tanto para a avaliação do equilíbrio funcional muscular, como também para a reabilitação das lesões do aparelho locomotor ${ }^{6}$. As articulações incluídas no exame são ombros, joelhos, tornozelos, quadris, cotovelos, punhos e também a coluna vertebral.

As vantagens existentes com esse método referem-se à resistência oferecida, que favorece o paciente trabalhar num valor submáximo ao arco de movimento doloroso e num valor máximo nas amplitudes não dolorosas; não há carga externa ao membro avaliado. É possível o desenvolvimento de velocidades de contração muscular mais rápidas semelhantes a algumas atividades esportivas, embora não atinjam velocidades como no saque de um tenista $\left(1.500^{\circ} / \mathrm{seg}\right)$ ou do arremessador de beisebol $\left(4.000^{\circ} / \mathrm{seg}\right)$; as mensurações são confiáveis, seguras, precisas, objetivas e reprodutíveis ${ }^{7,8}$.

As desvantagens estão ainda no preço elevado. Outro aspecto está no fato de que o aparelho isocinético não realiza o gesto ou o movimento específico de uma determinada modalidade esportiva. Portanto, o esforço realizado não envolve a energia cinética nas várias articulações, e sim numa única articulação, estando o restante do corpo sem deslocamento. $\mathrm{O}$ indivíduo realiza um teste ou fisioterapia monoarticular. Os movimentos são realizados normalmente sob a forma de exercício de cadeia cinética aberta.

As indicações para o exame referem-se ao estudo da proporção do equilíbrio muscular agonista/antagonista e na diferença entre os grupos musculares agonistas de um lado comparado ao seu lado contralateral. Avaliações pré-temporadas permitem determinação do adequado equilíbrio muscular. É possível avaliação do desempenho muscular do atleta sadio e frente a eventuais patologias do aparelho locomotor, sua recuperação pós-lesão ou possíveis seqüelas. O teste não tem como objetivo fornecer diagnóstico etiológico, mas permite adequada avaliação funcional do aparelho locomotor. Útil no estudo comparativo dos microtraumas de repetição e macrotraumas que ocasionaram lesões, tais como tendinopatias, entorses, rupturas musculotendíneas, contusões, fraturas, sinovites, miosites e síndromes neurovasculares. Será possível a orientação objetiva cinesioterápica em função do eventual déficit que o grupo muscular possa apresentar ${ }^{6}$. Nas afecções do joelho temos como interesse a avaliação nas lesões meniscais, ligamentares, capsulares, femoropatelares e musculotendíneas. É necessário um critério na indicação do exame. Havendo, portanto, patologia ou doença associada, torna-se prudente uma avaliação médica prévia, dando respaldo na responsabilidade para a indicação do exame.

Nas contra-indicações para sua realização encontram-se os acometimentos dolorosos com ou sem processo inflamatório clínico evidente, tempo insuficiente para um processo de reparação tecidual, e descompensações do sistema cardiorrespiratório, como hipertensão arterial não controlada, angina, arritmia. A limitação da amplitude de movimento torna-se uma contra-indicação relativa, pois não se obtém resultados ideais comparando-se lados com amplitudes de movimento diferentes.

\section{PARÂMETROS DE ANÁLISE}

\section{Pico de torque}

Representa o ponto de maior torque na amplitude de movimento; o torque ou momento de força representa o resultado da força aplicada num ponto multiplicada pela distância do ponto de aplicação dessa força ao centro de rotação do eixo de movimento, ou seja, $\mathrm{T}=\mathrm{F} \times \mathrm{d}$, medida em newton-metro $(\mathrm{Nm})$. O pico de torque pode, também, ser expresso pela percentagem do peso corporal do indivíduo, com o objetivo de comparar grupo de indivíduos. O torque e a velocidade angular de movimento são grandezas inversamente proporcionais, ou seja, quanto menor a velocidade angular realizada, maior será o torque; quanto maior a velocidade, menor o torque.

\section{Trabalho}

Representa a energia realizada no esforço muscular durante o movimento (produto do torque pelo deslocamento angular); é expresso em joule (J); existe o valor absoluto e em percentagem do peso corporal. Quanto menor a velocidade angular, maior o trabalho. 


\section{Potência}

É o resultado do trabalho realizado dividido pelo tempo, expresso em watt $(\mathrm{w})$. A velocidade angular é diretamente proporcional à potência, ou seja, quanto maior a velocidade angular, maior a potência; quanto menor a velocidade angular, menor a potência. Existe também o valor absoluto e o expresso em percentagem do peso corporal.

\section{Relação de equilíbrio agonista/antagonista}

É a divisão entre do valor do agonista e do antagonista, seja relacionado ao pico de torque, trabalho ou potência, expresso em percentagem. Portanto, representa a proporção entre tais grupos, existindo para cada articulação. Normalmente é avaliada nas velocidades angulares menores para o pico de torque e trabalho e nas velocidades angulares maiores para a potência. Mostra-se útil nos indivíduos que tiveram lesão do aparelho locomotor. Por exemplo, no joelho a relação entre o pico de torque dos flexores/extensores é por volta de $60 \%{ }^{5}$, ou seja, o valor absoluto do pico de torque dos flexores (numerador) dividido pelo dos extensores (denominador) resulta num valor em percentagem igual a 60. Portanto, a diferença entre os extensores (mais fortes) e os flexores (mais fracos) é de $40 \%$.

Existem outros parâmetros que podem ser avaliados, e que podem complementar a avaliação da função muscular:

Índice de resistência: obtido quando o número de repetições for igual ou superior a seis, mostrando-se a proporção (em percentagem) da metade final sobre a metade inicial do trabalho realizado; se o seu valor for, por exemplo, de $80 \%$, isto expressa que a $2^{\text {a }}$ metade das repetições representou um valor de $80 \%$ comparada à $1^{\text {a }}$ metade; logo, a diferença de $20 \%$ pode ser referida como índice de fadiga da metade final; representa energia que utiliza metabolismo anaeróbio.

Tempo de aceleração: tempo em segundos (normalmente décimos ou centésimos de segundo) necessário para que a velocidade angular preconizada seja atingida pelo esforço do indivíduo no início do movimento.

\section{APLICAÇÃO PRÁTICA}

O exame isocinético no joelho, assim como em outras articulações, seja para avaliação ou para reabilitação, pode utilizar velocidades angulares que variam, normalmente, entre $30 \%$ seg e $300 \%$ seg Tais velocidades podem ser consideradas lentas $\left(<180^{\circ} / \mathrm{seg}\right)$ ou rápidas $\left(>180^{\circ} / \mathrm{seg}\right)$. A velocidade de $180^{\circ} / \mathrm{seg}$ pode ser considerada intermediária. Para o melhor estudo do pico de torque e do trabalho, utiliza-se velocidade angular do tipo lenta, pois quanto menor a velocidade angular maior é o torque ou o trabalho. Neste caso, a velocidade mais usada é a de $60 \%$ seg. Já para a avaliação da potência, costuma-se usar velocidades de $180 \%$ seg a $300 \%$ seg, estando esta última voltada, principalmente, para os atletas de alto rendimento.

Portanto, num teste podemos ter:

- Velocidade angular: normalmente preconiza-se a utilização de uma velocidade lenta, que é indicada para a determinação do maior torque; velocidade rápida e/ou intermediária, enfocada para maior potência e índice de fadiga. Normalmente, realiza-se uma avaliação com até três velocidades diferentes, sendo necessária para cada pausa de 30 seg a 1 minuto. Na cinesioterapia é comum a utilização inicialmente de velocidades mais elevadas, pois são mais toleráveis e, portanto, menos dolorosas nos casos pós-lesão ou pós-operatórios. Não ocorrendo quadro doloroso ou inflamatório, objetivando o ganho da força muscular, diminui-se, então, a velocidade progressivamente, bem como aumenta-se o número de repetições.

- Número de repetições: na avaliação da função podemos realizar três a cinco repetições para as velocidades lentas ou intermediárias e 20 a 30 para as velocidades intermediárias ou rápidas; para a realização dos exercícios terapêuticos preconiza-se, normalmente, uma ou mais séries de até 10 sessões, entre cinco e 10 repetições, com velocidades predeterminadas.

- Tipo da contração: na avaliação é possível a utilização da contração nas formas concêntrica e/ou excêntrica. Normalmente é realizada a forma concêntrica, por ser mais segura e de fácil compreensão para a execução do atleta. Existem aparelhos que realizam, também, a forma ativa assistida, passiva e isométrica (velocidade angular $=$ zero). Nesse caso, consiste num método auxiliar na cinesioterapia nos indivíduos que se encontram, ainda, com limitação da amplitude de movimento e presença de dor.

Orientações gerais: para a adequada realização e compreensão do teste, o indivíduo deve fazer um aquecimento prévio numa bicicleta ou esteira ergométrica por cinco minutos; é importante a adaptação aos movimentos através da sua realização com esforço submáximo; o corpo, que fica numa posição sentada na avaliação do joelho, deve estar bem estabilizado; o procedimento deve ser bem explicado e, inclusive, estimulado durante a execução, para um melhor aproveitamento.

\section{INTERPRETAÇÃO}

A relação entre agonista/antagonista é uma forma adequada para saber se existe proporção e conseqüentemente o equilíbrio muscular. Na literatura, os relatos a respeito da relação flexora/extensora nos joelhos sem lesão variam normalmente entre $55 \%$ e $77 \% 9,10$. Foi descrito que joelhos dominantes sem lesão de jogadores de futebol americano apresentavam relação de $67 \%$ e $82 \%$ nas velocidades, res- 
pectivamente, de $90 \% \mathrm{seg}$ e $300^{\circ} / \mathrm{seg}^{11}$. A diferença entre o lado dominante e não dominante não foi significativa. Foi mostrado que, nos indivíduos com insuficiência de ligamento cruzado anterior (LCA) com tempo médio de evolução de 2,7 anos, a relação flexora/extensora era de $66 \%$ e $82 \%$ nas velocidades, respectivamente, de $60^{\circ} \%$ seg e $180^{\circ} \%$ $\mathrm{seg}^{10}$. Alguns estudos mostraram que ocorre desequilíbrio muscular, com déficit de força da musculatura do joelho com insuficiência de LCA $^{12-14}$.

Um estudo realizado com atletas com ruptura total de LCA num dos joelhos mostrou valores do equilíbrio flexor/ extensor semelhantes na velocidade de $60 \%$ seg entre os lados acometido (60\%) e não acometido (57\%), apesar da ruptura completa do $\mathrm{LCA}^{5}$. O mesmo não ocorreu na velocidade rápida de $240 \% \mathrm{seg}$, em que o lado acometido mostrou relação de $75 \%$ e o lado não acometido $65 \%$, havendo diferença estatisticamente significante. Portanto, à medida que ocorreu aumento para esta velocidade, ocorreu também um aumento da relação flexora/extensora do joelho, indicando leve tendência do grupo muscular flexor se aproximar do extensor, mais pronunciada no lado com lesão. No entanto, nos indivíduos que realizaram reabilitação e atingiram graus adequados de equilíbrio muscular, existe a possibilidade de ocorrer episódios de falseios e perpetuação da instabilidade em joelhos com LCA insuficiente ${ }^{10,12}$. Para os esportistas que necessitam ser submetidos à reconstrução do LCA, pois é a forma que há para se ter novamente um joelho estável e em condições adequadas para realizar prática de esporte competitivo, o teste com aparelho isocinético poderá servir como parâmetro pré-operatório para avaliação do equilíbrio muscular, bem como na sua evolução durante o período da reabilitação pós-operatória. No nosso laboratório o teste isocinético para os casos de pósoperatório de LCA realizado com enxerto do tendão patelar é indicado no 4ํㅡㄹ. A possibilidade da execução do exame isocinético deve ser considerada nos indivíduos atletas, mesmo apresentando diagnóstico de ruptura do LCA, lesão meniscal ou acometimentos femoropatelares, desde

\section{REFERÊNCIAS}

1. Hislop HJ, Perrine JJ. The isokinetic concept of exercise. Phys Ther 1967;47:114-7.

2. Greve JMD, Terreri AST, Plapler PG. Avaliação do torque isocinético flexor e extensor do tronco em atletas e sedentários normais. Rev Hosp Clin Fac Med S Paulo 1997;52:154-7.

3. Nery CAS, Alloza JFM, Laurino CFS, Tanaka GS. Avaliação da força muscular isocinética do pé e tornozelo após tratamento cirúrgico das lesões do tendão de Aquiles, utilizando a transferência do tendão fibular curto. Rev Bras Ortop 1997;32:503-12.

4. Shinzato GT, Vasconcelos JCP, Ogawa CT, Sampaio ICSP, Gonçalves A, Neves EM. Protocolo de avaliação funcional de joelho em patologias ortopédicas. Acta Fisiátrica 1996;3:30-6. que o médico indique e que não exista impedimento do exame, principalmente devido a dor, bloqueios ou limitação da amplitude de movimento. Estudos de avaliações isocinéticas nos pós-operatórios de LCA mostraram no lado operado valores que podem ser iguais ou apresentar um déficit pequeno comparado ao lado não operado ${ }^{12,15,16}$. Em alguns casos, ocorreu déficit em algum ângulo de movimento específico, tanto para o quadríceps $\left(30^{\circ}\right.$ e $60^{\circ}$ de flexão), como para os isquiotibiais $\left(30^{\circ} \text { de flexão }\right)^{12}$.

$\mathrm{Na}$ interpretação dos resultados admite-se que o valor de um grupo muscular sem acometimento pode ser considerado normal, desde que seja igual ou apresente diferença de até $10 \%$ comparado ao grupo muscular contralateral. Para a realização das atividades esportivas, diferenças de até $20 \%$ podem ser aceitas. Nesse caso, pode ser utilizado para servir como parâmetro dentro dos critérios de retorno a atividade esportiva ${ }^{17}$. Deve ser respeitado o valor absoluto no que se refere ao sexo, pois, normalmente, o sexo masculino apresenta torque $40 \%$ a $50 \%$ maior. A proporção agonista/antagonista tende a ser semelhante ${ }^{18}$.

A atividade esportiva e o nível de condicionamento físico podem constituir fatores determinantes na adequada interpretação dos resultados.

\section{CONCLUSÃO}

É na avaliação funcional e na reabilitação do joelho, como em todo aparelho locomotor, que o aparelho isocinético apresenta seu ponto estratégico de aplicação, constituindo-se num importante método auxiliar para tal investigação. Nesse contexto, o equilíbrio muscular agonista/antagonista, a comparação entre os lados direito e esquerdo e a necessidade do retorno adequado a atividade esportiva póslesão ou no pós-operatório, com níveis semelhantes ou superiores de condicionamento e aptidão física, são objetivos do atleta e de sua equipe multidisciplinar para serem atingidos. Eventuais déficits musculares poderão ser avaliados e quantificados de forma objetiva, facilitando e orientando o valioso trabalho do reequilíbrio muscular.

5. Terreri AS, Ambrósio MA, Pedrinelli A, Albuquerque RFM, Andrusaitis F, Greve JMD, Carazzato JG, Amatuzzi MM. Isokinetic assessment of the flexor-extensor balance in athletes with total rupture of the anterior cruciate ligament. Rev Hosp Clin Fac Med S Paulo 1999;54:53-60.

6. Puhl W, Noack W, Scharf HP, Sedunko F. Isokinetisches Muskeltraining in Sport und Rehabilitation. Perimed Fachbuch - Verlagsgesellschaft mbH. Erlangen, 1988.

7. Davies GJ. A compendium of isokinetics in clinical usage and rehabilitation techniques, $4^{\text {th }}$ ed. Onalaska, WI S \& Publishers, 1992

8. Osternig LR. Isokinetic dinamometry: implications for muscle testing and rehabilitation. Exerc Sport Sci Ver 1986;14:45-80. 
9. Grace TG, Sweetser, ER, Nelson MA, Ydens LR, Skipper BJ. Isokinetic muscle imbalance and knee joint injuries. J Bone Joint Surg [Am] 1984;66:734-40.

10. Harilainen A, Alaranta H, Sandelin J, Vanhanen I. Good muscle performance does not compensate instability symptoms in chronic anterior cruciate ligament deficiency. Knee Surg Sports Traumatol Arthroscopy 1995;3:135-7.

11. Stafford MG, Grana WA. Hamstrings/quadriceps ratios in college football players: A high velocity evaluation. Am J Sports Med 1984;12:20911.

12. Murray SM, Warren RF, Otis JC, Kroll M, Wickiewicz TL. Torque-velocity relationships of the knee extensor and flexor muscles in individuals sustaining injuries of the anterior cruciate ligament. Am J Sports Med 1984;12:436-40.

13. Shirakura K, Kato K, Udagawa E. Characteristics of the isokinetic performance of patients with injured cruciate ligaments. Am J Sports Med 1992;20:754-60.
14. Tibone JE, Antich TJ, Fanton GS, Moynes DR, Perry J. Functional analysis of anterior cruciate ligament instability. Am J Sports Med 1988;14: 276-84.

15. Seto JL, Orofino AS, Morrissey MC, Medeiros JM, Mason WJ. Assessment of quadriceps/hamstring strength, knee ligament stability, functional and sports activity levels five years after anterior cruciate ligament reconstruction. Am J Sports Med 1988;16:170-80.

16. Yasuda K, Ohkoshi Y, Tanabe Y, Kaneda Kiyoshi. Quantitative evaluation of knee instability and muscle strength after anterior cruciate ligament reconstruction using patellar and quadriceps tendon. Am J Sports Med 1992;20:471-5

17. Reid DC. Sports injury, assessment and rehabilitation. Churchill Livingstone, New York, 1992.

18. Frontera WR, Hughes VA, Dallal GE, Evans WJ. Reliability of isokinetic muscle strength testing in 45 to 78 year old men and women. Arch Phys Med Rehabil 1993;74:1181-5. 